\title{
Generation of a Turbulent Boundary Layer Inflow for RANS Simulations
}

\author{
A. Rona, M. Grottadaurea†, M. Monti ${ }^{\ddagger}$ \\ University of Leicester, Leicester LE1 7RH, United Kingdom \\ C. Airiau ${ }^{a, b \S}$ T. Gandhi ${ }^{a, b \uparrow}$ \\ ${ }^{a}$ Université de Toulouse; INPT, UPS; IMFT (Institut de Méchanique des Fluides de Toulouse) \\ Allée Camille Soula, F-31400 Toulouse, France \\ ${ }^{b}$ CNRS; IMFT; F-31400 Toulouse, France
}

The generation of a fully turbulent boundary layer profile is investigated using analytical and numerical methods over the Reynolds number range $300 \leq R e_{\theta} \leq 31000$. The predictions are validated against reference wind tunnel measurements under zero streamwise pressure gradient. The analytical method is then tested for favourable pressure gradient by modelling the turbulent boundary layer approaching a two-dimensional potential sink. Both methods show a good predictive ability under a zero pressure gradient, with the numerical method providing a complete velocity profile through the laminar sub-layer down to the wall. This work is of practical interest to computational fluid dynamic practitioners for generating an equilibrium thick turbulent boundary layer at the computational domain inflow.

\section{Nomenclature}

Integration constant for the outer region velocity profile

Logarithmic law constant

Logarithmic law constant for the inner region

Non-dimensional momentum thickness

Skin friction coefficient

Normalised mixing length at $y \rightarrow \delta$

Logarithmic law constant for the outer region

Van Driest near wall damping correction

Normalised defect velocity, $u_{e}^{+}-u^{+}$

Shape factor

Sink flow acceleration parameter

Flat plate length

Mixing length

Number of data points in the experimental velocity profile

Pressure

Potential sink strength

Real number

Reynolds number

Temperature

Tangential velocity

\footnotetext{
${ }^{*}$ Lecturer, Department of Engineering, AIAA Member.

${ }^{\dagger}$ Researcher, Department of Engineering.

${ }^{\ddagger}$ Researcher, Department of Engineering.

$\S$ Professor.

I Doctorant, IMFT.
} 


\begin{tabular}{|c|c|}
\hline$u_{\tau}$ & Friction velocity \\
\hline$x$ & Tangential distance from the boundary layer leading edge \\
\hline$y$ & Wall-normal distance from the solid wall \\
\hline$\alpha$ & Outer region normalised displacement thickness \\
\hline$\beta$ & Normalised streamwise pressure gradient \\
\hline$\tilde{\beta}$ & Non-dimensional free-stream acceleration parameter, $\tilde{\beta}=2 \beta F_{1}$ \\
\hline$\beta_{c}$ & Clauser parameter \\
\hline$\delta$ & Boundary layer thickness \\
\hline$\delta^{*}$ & Boundary layer displacement thickness \\
\hline$\epsilon$ & Least squares error \\
\hline$\eta$ & Outer layer non-dimensional coordinate, $\eta=y / \delta$ \\
\hline$\theta$ & Boundary layer momentum thickness \\
\hline$\kappa$ & von Karman constant, $\kappa=0.41$ \\
\hline$\lambda$ & Local free-stream to leading edge free-stream velocity ratio, $u_{e} / u_{0}$ \\
\hline$\mu_{l}$ & Laminar or molecular viscosity \\
\hline$\nu$ & Kinematic viscosity \\
\hline$\Pi$ & Wake parameter \\
\hline$\rho$ & Density \\
\hline$\tau$ & Tangential shear stress \\
\hline \multicolumn{2}{|c|}{ Subscripts } \\
\hline$a$ & Analytical prediction \\
\hline$e$ & Free-stream condition \\
\hline$e x$ & Experimental value \\
\hline$l$ & Laminar component \\
\hline$t$ & Reynolds average (turbulent) component \\
\hline$w$ & Wall condition \\
\hline 0 & Leading edge condition \\
\hline \multicolumn{2}{|c|}{ Superscripts } \\
\hline+ & Inner layer scaling \\
\hline$\overline{()}$ & Time average \\
\hline 1 & Fluctuation about the time-mean value \\
\hline
\end{tabular}

\section{Introduction}

Computational fluid dynamic simulations of wall bounded flows, such as the flow over high-lift devices, ailerons, the elevators and the rudder, often use a turbulent boundary layer inflow to reduce the computational domain size with respect to a full wing, tailplane or fin simulation. The quality of the numerical predictions can be significantly affected by how well the boundary layer inflow is modelled. This paper compares the use of analytical correlations and of an auxiliary boundary layer numerical method to generate a turbulent boundary layer inflow for CFD over a wide Reynolds number range.

Computational fluid dynamic simulations of individual airframe components are commonly used to study the local aerodynamics in details. ${ }^{1-3}$ This enables to achieve a sufficient level of spatial and temporal refinement around the specific components to model the onset of self-sustained oscillations, such as those in cavity flows, edge tones and other fluid-resonant geometries. These flow instabilities contribute to airframe noise and a good quality inflow prediction is very important to achieve quantitative predictions of the radiated noise pattern. For instance, in a cavity, the inflow momentum thickness has a direct influence on acoustic mode selection. ${ }^{4}$

Where the inflow features a fully developed turbulent boundary layer, an analytical profile for the mean velocity can be imposed, derived from the integral boundary layer parameters as determined from either a larger-scale numerical simulation or from experiment. A common choice for specifying the boundary layer inflow in aerodynamics is by defining the inflow free-stream velocity $u_{e}$, temperature $T_{e}$, pressure $p_{e}$, boundary layer thickness $\delta$, momentum thickness based Reynolds number $R e_{\theta}$, shape factor $H$, and the streamwise pressure gradient $d p_{e} / d x$.

An alternative approach to define the mean velocity inflow is by using an auxiliary numerical simulation of the upstream boundary layer obtained, for instance, from running two-dimensional companion software 
by Wilcox. ${ }^{5}$

This paper presents and validates one analytical and one numerical approach for generating a turbulent boundary layer inflow in CFD. The analytical method is a variant of the defect law by Coles ${ }^{6}{ }^{6}$ while the numerical method is derived from matched asymptotic expansions. ${ }^{7}$ The analytical approach is then applied to a boundary layer with favourable pressure gradient, in which a two-dimensional potential sink is used to generate the outer flow.

Section II details the analytical method used to generate the outer layer velocity profile in a turbulent boundary layer. Section III details the numerical method based on the equilibrium boundary layer model. Section IV validates both methods using zero pressure gradient velocity data over the Reynolds number range $300 \leq R e_{\theta} \leq 31000$. Section $V$ presents a two-dimensional sink flow and extends the validation to the favourable pressure gradient boundary layer approaching the sink.

\section{Analytical method}

To describe the mean velocity profile in a turbulent boundary layer, similarity solutions are sought in the inner and the outer regions. In the inner region, the mean streamwise velocity $u$ scales with the wall friction velocity $u_{\tau}$ and with the viscous length scale $l=\nu_{l} / u_{\tau}$, so that

$$
\frac{u}{u_{\tau}}=f\left(y^{+}\right)
$$

where $y^{+}=y u_{\tau} / \nu_{l}$ is the inner scaling non-dimensional wall-normal distance. In outer region, the velocity profile is described by the velocity defect law

$$
\frac{u_{e}-u}{u_{\tau}}=f(\eta)
$$

where $\eta=y / \delta$ is the outer scaling non-dimensional wall-normal distance, $u_{e}$ is the free-stream velocity, $\nu_{l}$ is the laminar kinematic viscosity, $y$ is the wall-normal distance and $\delta$ is the boundary layer thickness, which is taken as the wall-normal distance at which $u=u_{e}$.

Based on the existence of an overlap region between the inner and the outer regions, Coles ${ }^{6}$ proposed the following additive law of the wall and law of the wake in non-dimensional form:

$$
\begin{array}{r}
u^{+}=\frac{1}{\kappa} \ln y^{+}+B+\frac{\Pi}{\kappa} f(\eta) \\
f(\eta)=1-\cos (\pi \eta)
\end{array}
$$

where $u^{+}=u / u_{\tau}$ is the normalized streamwise velocity, $\Pi$ is the wake parameter, $\kappa$ the von Kármán constant, and $B$ the logarithmic law constant.

Coles $^{6}$ determined the wake parameter as

$$
\Pi=\kappa / 2\left(u_{e}^{+}-\kappa^{-1} \ln R e_{\tau}-B\right)
$$

where $R e_{\tau}=\delta u_{\tau} / \nu_{l}$ is the boundary layer Reynolds number and $u_{e}^{+}=u_{e} / u_{\tau}$ is the normalized free-stream velocity.

Let

$$
f(\eta)=A_{1} \eta^{2}+A_{2} \eta^{3}
$$

be a cubic polynomial approximation to $f(\eta)$ in eq. 3. Substituting the boundary conditions

$$
\left.u\right|_{y=\delta}=u_{e}
$$

and

$$
\left.\frac{\partial u}{\partial y}\right|_{y=\delta}=0
$$

in eq. 3 , with $f(\eta)$ from eq. 5 , gives $A_{1}=6[1+1 /(6 \Pi)]$ and $A_{2}=-4[1+1 /(4 \Pi)]$, with $\Pi$ defined by eq. 4 . The law of the wake of eq. 3 then becomes

$$
u^{+}=\underbrace{\overbrace{\frac{1}{\kappa} \ln y^{+}+B}^{\text {Log-law of the wall }}+\frac{1}{k} \eta^{2}(1-\eta)}_{\text {Pure wall flow }}+\underbrace{+2 \frac{\Pi}{\kappa} \eta^{2}(3-2 \eta)}_{\text {Pure wake component }}
$$


Equation 8 is validated over a relatively wide range of momentum thickness based Reynolds number $R e_{\theta}=$ $u_{e} \theta / \nu_{l}$ in section IV. To evaluate eq. 8 , the authors take $\kappa=0.41$ and $B=5.0$, as proposed by Coles. ${ }^{6}$

\section{Successive complementary expansion method}

The successive complementary expansion method consists in seeking contiguous asymptotic matches between the inner and the outer regions of an incompressible turbulent boundary layer. This approach is detailed in Cousteix \& Mauss ${ }^{7}$ and this paper only reproduces the key steps that support the authors' application to turbulent boundary layers.

\section{III.A. Mixing length model}

Across the boundary layer, the local shear stress

$$
\tau=\mu_{l} \frac{\partial u}{\partial y}-\rho \overline{u^{\prime} v^{\prime}}=\tau_{l}+\tau_{t}
$$

where $u^{\prime}$ and $v^{\prime}$ are the time-dependent fluctuations of the streamwise and flow-normal velocity components and are unknown. To avoid having to resolve these unknowns, the Reynolds shear stress $\tau_{t}$ is evaluated using Prandtl's mixing length model, ${ }^{8}$ with the Van Driest ${ }^{9}$ near-wall damping correction $\tilde{\mathrm{F}}$. This gives

$$
\tau_{t}=\rho \tilde{\mathrm{F}}^{2} \ell^{2}\left|\frac{\partial u}{\partial y}\right|\left(\frac{\partial u}{\partial y}\right)
$$

where $\tilde{\mathrm{F}}=1-\exp \left(-y^{+} / 26\right)$.

In the inner region, $\ell=\kappa y$, while in the outer region, $\ell / \delta \rightarrow c_{\ell}$ as $y \rightarrow \delta$ and $c_{\ell}=0.085$. These two trends can be merged analytically into a single distribution for the mixing length $\ell$ across the full boundary layer by the use of a blending function. Michel et al. ${ }^{10}$ used the blending function

$$
\ell(\eta)=\delta c_{\ell} \tanh \left(\frac{\kappa \eta}{c_{\ell}}\right)
$$

with $\kappa=0.41$. The authors propose an alternative blending function that is shown in section IV to give an improved prediction of the turbulent shear stress profile at the interface between the inner and the outer layer, at low Reynolds numbers $R e_{\tau}$. This is

$$
\ell(\eta)=\delta \frac{\kappa \eta}{\left[1+\left(\kappa \eta / c_{\ell}\right)^{n}\right]^{1 / n}}
$$

For $2.6<n<2.7$, the $\ell(\eta)$ profile from equation 12 almost matches that from equation 11.

\section{III.B. Inner region velocity profile}

Normalising the local shear stress $\tau$ in eq. 10 by $\rho u_{\tau}^{2}$ and assuming a monotonic velocity profile gives

$$
\frac{\tau}{\tau_{w}}=\frac{\partial u^{+}}{\partial y^{+}}+\ell^{+2} \tilde{\mathrm{F}}^{2}\left(\frac{\partial u^{+}}{\partial y^{+}}\right)^{2}
$$

where $\ell^{+}=\ell u_{\tau} / \nu_{l}$. In the limit $y^{+} \rightarrow 0, \tau \rightarrow \tau_{w}$ and eq. 13 becomes

$$
1=\frac{\partial u^{+}}{\partial y^{+}}+\ell^{+2} \tilde{\mathrm{F}}^{2}\left(\frac{\partial u^{+}}{\partial y^{+}}\right)^{2}
$$

Equation 14 is a quadratic in $\partial u^{+} / \partial y^{+}$with $\operatorname{root}^{7}$

$$
\frac{\partial u^{+}}{\partial y^{+}}=\frac{2}{1+\sqrt{1+4\left[\ell^{+}\left(y^{+}\right) \tilde{\mathrm{F}}\left(y^{+}\right)\right]^{2}}}
$$

Integrating equation 15 with respect to $y^{+}$with the boundary condition $u^{+}(x, 0)=0$ gives the inner layer tangential velocity profile that asymptotes to the log-law of the wall in equation 8 for $y^{+}>80$ with $B=5.28$. 


\section{III.C. Outer region velocity profile}

In an equilibrium turbulent boundary layer, a similarity solution for the outer layer is sought in terms of the velocity defect $F^{\prime}(\eta)=u_{e}^{+}-u^{+}$, under the local assumption of no boundary layer growth. Expressing $\tau / \tau_{w}$ as a function of $F$ and $\eta$ gives $^{7}$

$$
\frac{\tau}{\tau_{w}}=1-\frac{F}{F_{1}}+\left(\frac{1}{F_{1}}+2 \beta\right) \eta F^{\prime}
$$

where

$$
F=\int_{0}^{\eta} F^{\prime}(\xi) d \xi ; \quad F_{1}=F(1) ; \quad \beta=-\frac{\delta}{u_{\tau}} \frac{d u_{e}}{d x}
$$

In the outer region, the Reynolds stress component is dominant over the laminar shear stress, so $\tau \simeq \tau_{t}$. From eq. 10, noting that the Van Driest damping constant $\tilde{\mathrm{F}} \rightarrow 1$ at $y^{+} \geq 100, \tau / \tau_{w}=(\ell / \delta)^{2} F^{\prime \prime 2}$, where $F^{\prime \prime}=d F^{\prime} / d \eta$. Substituting for $\tau / \tau_{w}$ in eq. 16, the similarity solution for the outer region becomes

$$
\left(\frac{\ell}{\delta}\right)^{2} F^{\prime \prime 2}=1-\frac{F}{F_{1}}+\left(\frac{1}{F_{1}}+2 \beta\right) \eta F^{\prime}
$$

\section{III.D. Asymptotic matching of the inner and outer profiles}

A matching condition is sought for the velocity profiles of the inner and outer regions, equations 15 and 18 . This is obtained from standard asymptotic analysis ${ }^{7}$ by considering eq. 15 in the limit $y^{+} \rightarrow \infty$ and eq. 18 in the limit $\eta \rightarrow 0$ that give respectively ${ }^{7}$

$$
\begin{aligned}
u^{+} & =\kappa^{-1} \ln y^{+}+C \\
u_{e}^{+}-u^{+} & =-\kappa^{-1} \ln \eta+D_{v}
\end{aligned}
$$

Adding eq. 19 to eq. 20 gives $^{7}$

$$
u_{e}^{+}=\kappa^{-1} \ln \frac{u_{\tau} \delta}{\nu_{l}}+C+D_{v}
$$

Equation 21 can be re-cast as function of the wall skin friction coefficient $C_{f}=\tau_{w} /\left(0.5 \rho u_{e}^{2}\right)$ that is imposed as equal in the inner and outer regions and provides the matching criterion for the two profiles at $R e_{\tau}=u_{\tau} \delta / \nu_{l}$

$$
\sqrt{\frac{2}{C_{f}}}=\kappa^{-1} \ln R e_{\tau}+C+D_{v}
$$

\section{III.E. Numerical implementation}

Expliciting the outer region velocity profile poses several challenges. Equation 18 is non-linear and is illdefined at the upper boundary layer limit, at $\eta \rightarrow 1$, where $F^{\prime \prime} \rightarrow 0$, and at the lower boundary layer limit, at $\eta \rightarrow 0$, where $\ell / \delta \rightarrow 0$ and $F^{\prime \prime} \rightarrow \infty$. To solve the problem, auxiliary approximate solutions are imposed on the floor of the laminar sub-layer and at the edge of the boundary layer, as shown in figure 1, so that the edges of the inner and of the outer regions are modelled analytically while the overlap region is resolved numerically.

Let $f(\eta)=F(\eta) / F(1)$. On the floor of the laminar sub-layer, imposing $\eta=0$ and $\ell=\kappa y$, as in section III.A, eq. 18 becomes

$$
\left[\kappa \eta F_{1} f^{\prime \prime}(\eta)\right]^{2}=1-f(\eta)+\left(1+2 \beta F_{1}\right) \eta f^{\prime}(\eta)
$$

with the boundary condition $f(0)=0$. Let $\tilde{\beta}=2 \beta F_{1}$. In a zero pressure gradient boundary layer, $\beta=0$ by eq. 17 , for which eq. 23 has the explicit solution

$$
f(\eta)=\frac{\eta^{2}}{4 \alpha^{2}}-\frac{\eta \ln \eta}{\alpha}+A \eta ; \quad f^{\prime}(\eta)=\frac{\eta}{2 \alpha^{2}}-\frac{1+\log \eta}{\alpha}+A ; \quad f^{\prime \prime}(\eta)=\frac{1}{2 \alpha^{2}}-\frac{1}{\alpha \eta}
$$

with $\alpha=F_{1} \kappa$. The integration constant $A$ is determined by evaluating $f^{\prime}(\eta)$ at $\eta=\epsilon_{0}$ on the floor of the laminar sub-layer. In a non-zero pressure gradient boundary layer, $\tilde{\beta} \eta f^{\prime} \rightarrow 0$ as $\eta \rightarrow 0$, so the zero pressure gradient profile is used on the floor of the laminar sub-layer for all $\beta$. 


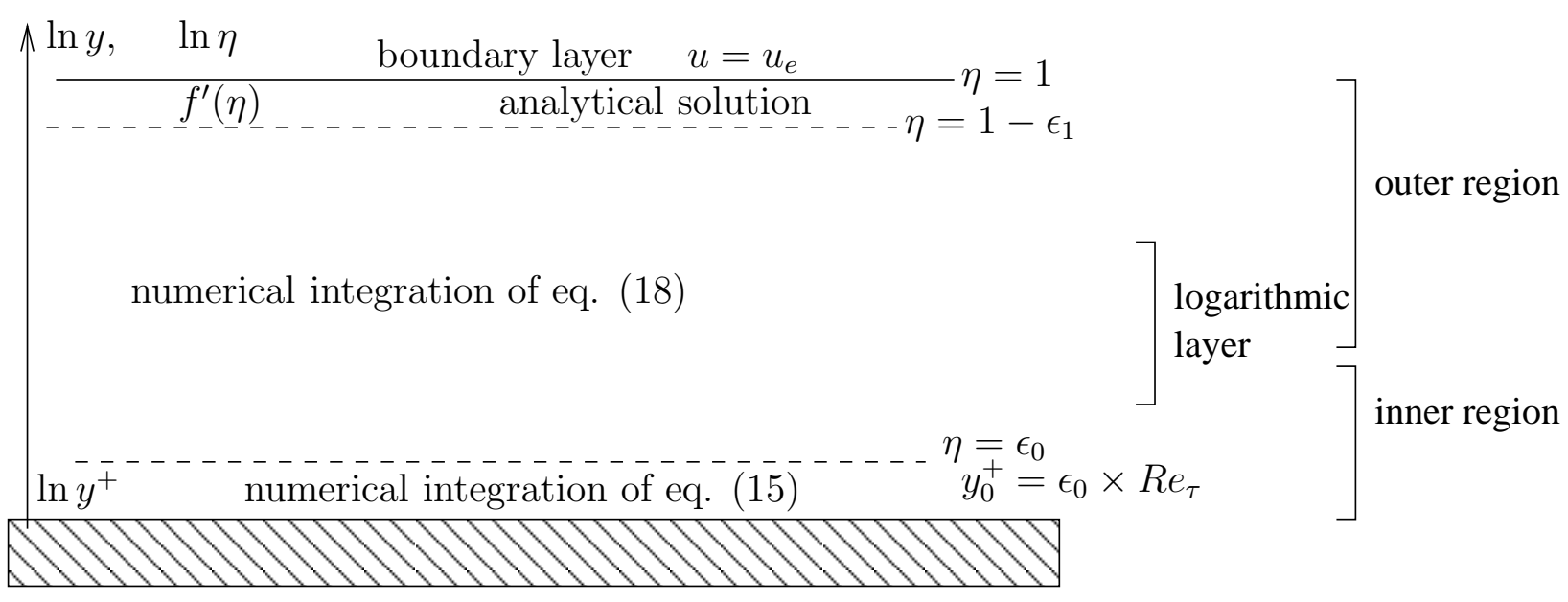

Figure 1. Boundary layer decks.

At the edge of the boundary layer, at $\eta=1$, eq. 18 becomes

$$
\left[\ell_{1} F_{1} f^{\prime \prime}(\eta)\right]^{2}=1-f(\eta)+(1+\tilde{\beta}) \eta f^{\prime}(\eta)
$$

with the boundary conditions $f(1)=1, f^{\prime}(1)=0, f^{\prime \prime}(1)=0$ and $\ell_{1}$ evaluated from eq. 12 at $\eta=1$. Cousteix ${ }^{11}$ proposed the solution for eq. 24

$$
f(\eta)=1-\frac{(1-\eta)^{3}}{3} ; \quad f^{\prime}(\eta)=(1-\eta)^{2} ; \quad f^{\prime \prime}(\eta)=-2+2 \eta
$$

for $\beta=0$, that has the attractive property of being independent from $F_{1}$ and $\ell_{1}$ and is the solution used in this work. The same solution is used for $\beta \neq 0$, as $\tilde{\beta} \eta f^{\prime}(\eta)=0$ by the boundary condition $f^{\prime}(1)=0$ in eq. 24 .

\section{Zero pressure gradient boundary layers}

The analytical and numerical methods for predicting a boundary layer mean turbulent velocity profile are tested against a range of streamwise velocity measurements of zero pressure gradient boundary layers ${ }^{12-15}$ over the range $300 \leq R e_{\theta} \leq 31000$. Table 1 lists the values of $u_{e}^{+}, R e_{\tau}$ and $\Pi$ at each $R e_{\theta}$ of the experimental velocity traverse records. ${ }^{12-15}$ The values of $u_{e}^{+}$and $R e_{\tau}$ are the ones reported in experiment ${ }^{12-15}$ while $\Pi$ has been obtained by fitting eq. 8 using the least squares fit.

\begin{tabular}{|c|c|c|c|c|c||c|c|c|}
\hline$R e_{\theta}$ & $R e_{\tau}$ & $u_{e}^{+}$ & $\Pi$ & $100 \times \epsilon$ & Symbol & $\left(R e_{\tau}\right)_{\text {num }}$ & $\left(u_{e}^{+}\right)_{\text {num }}$ & $100 \times \epsilon_{\text {num }}$ \\
\hline 300 & 145 & 18.25 & 0.228 & 1.33 & $\circ$ & 142 & 18.54 & 2.12 \\
697 & 335 & 20.25 & 0.219 & 1.35 & $*$ & 315 & 20.77 & 3.31 \\
1003 & 460 & 21.5 & 0.317 & 1.78 & $\triangle$ & 446 & 21.66 & 2.39 \\
1430 & 640 & 22.4 & 0.336 & 1.38 & $\cdot$ & 627 & 22.51 & 2.77 \\
2900 & 1192 & 24.33 & 0.421 & 1.02 & $\triangleleft$ & 1240 & 24.17 & 2.48 \\
3654 & 1365 & 25.38 & 0.568 & 0.72 & $\times$ & 1551 & 24.71 & 2.44 \\
5200 & 2000 & 26 & 0.505 & 1.62 & $\triangleright$ & 2185 & 25.54 & 2.38 \\
12633 & 4436 & 28.62 & 0.643 & 0.71 & $\square$ & 5188 & 27.65 & 2.51 \\
13000 & 4770 & 28 & 0.480 & 0.99 & $\diamond$ & 5335 & 27.72 & 1.84 \\
22845 & 8000 & 30.15 & 0.662 & 1.01 & + & 9258 & 29.06 & 2.34 \\
31000 & 13030 & 30 & 0.388 & 2.05 & $\star$ & 12845 & 29.79 & 1.86 \\
\hline
\end{tabular}

Table 1. Experimental velocity profiles.

The normalized mean streamwise velocity $u^{+}$is plotted against the normalized wall-normal distance $y^{+}$ in figure 8 for different Reynolds numbers. The symbols used in figure 8 are measured values ${ }^{12-15}$ at different 
$R e_{\theta}$, labelled as in table 1 . The continuous lines show the fitted analytical profiles for the outer layer. For clarity, an incremental shift of $u^{+}=2.5$ is applied to all curves. The three 0 labels on the vertical axis of figure 2 correspond to $R e_{\theta}=300, R e_{\theta}=5200$, and $R e_{\theta}=31000$ respectively.

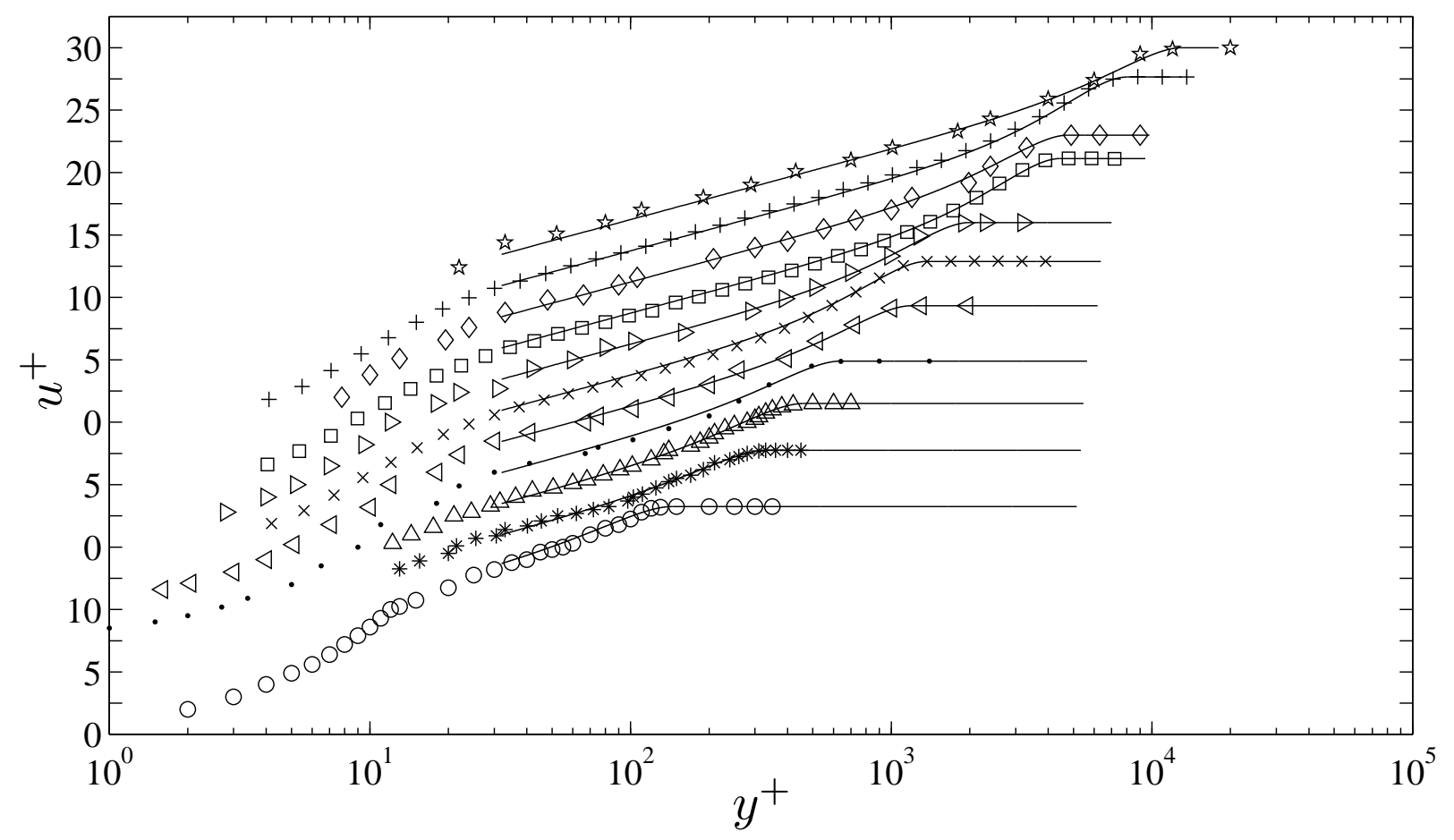

Figure 2. Turbulent boundary layer profiles fitted to eq. 8. Symbols as in table 1.

The quality of the predictions is quantified by evaluating the mean square percentage error $\epsilon$ for each profile

$$
\epsilon=\sqrt{\frac{1}{N} \sum_{i=1}^{N}\left(\frac{u_{a}^{+}-u_{e x}^{+}}{u_{e x}^{+}}\right)^{2}}
$$

where $u_{a}^{+}$is the predicted value and $u_{e x}^{+}$is the corresponding experimental value for a given $y_{i}^{+}$in a discretized velocity profile of $N$ points. The mean square percentage error $\epsilon$ obtained at different $R e_{\theta}$ with $u_{a}^{+}$evaluated from equation 8 is reported in table 1 . The maximum $\epsilon$ is $2.05 \%$ at $R e_{\theta}=31000$. Such error enables the use of eq. 8 to predict the mean streamwise velocity of boundary layers in many common engineering applications, where an error margin of $5 \%$ is often acceptable. The experimental data seem to be randomly distributed about the fitted curve with no underlying trend, suggesting that the curve fit has captured most of the $u^{+}$dependence on $\delta, u_{e}, u_{\tau}$, and $R e_{\theta}$.

Figure 3 compares velocity profiles obtained using the successive complementary expansion method of section III against the same experimental data of figure 2. In this application of the successive complementary expansion method, $n=4$ was used for the numerical prediction of the mixing length in eq. 12 . The symbols used in figure 2 are measured values ${ }^{12-15}$ at different $R e_{\theta}$, labelled as in table 1 . The continuous lines show the normalized numerical velocity profiles. For clarity, the same incremental shift of $u^{+}=2.5$ as in figure 2 is applied to all curves. The origin of the ordinate of figure 3 refers to the $R e_{\theta}=300$ profile. Figure 3 shows that the complementary expansion method of section III produces a full velocity profile down to the wall. In the outer layer, the complementary expansion method captures the Reynolds number dependent transition between the log-law and the constant free-stream velocity for most of the curves. The free-stream velocity at $R e_{\theta}=22845,12663$ and 3654 appear to be under-predicted. This is confirmed by the corresponding numerical mean square percentage error, $\epsilon_{n u m}$, which is computed by evaluating $u_{a}^{+}$in eq. 26 using the successive complementary expansion method. The $\epsilon_{n u m}$ at $R e_{\theta}=22845,12663$ and 3654 are higher than at some of the other Reynolds numbers, due to the difference in the normalized free-stream velocity between experiment and prediction. Whereas, in general, the error from the numerical velocity profile is higher than 
that from the analytical profile, it is within the range for which the predictions can be used for engineering accurate predictions.

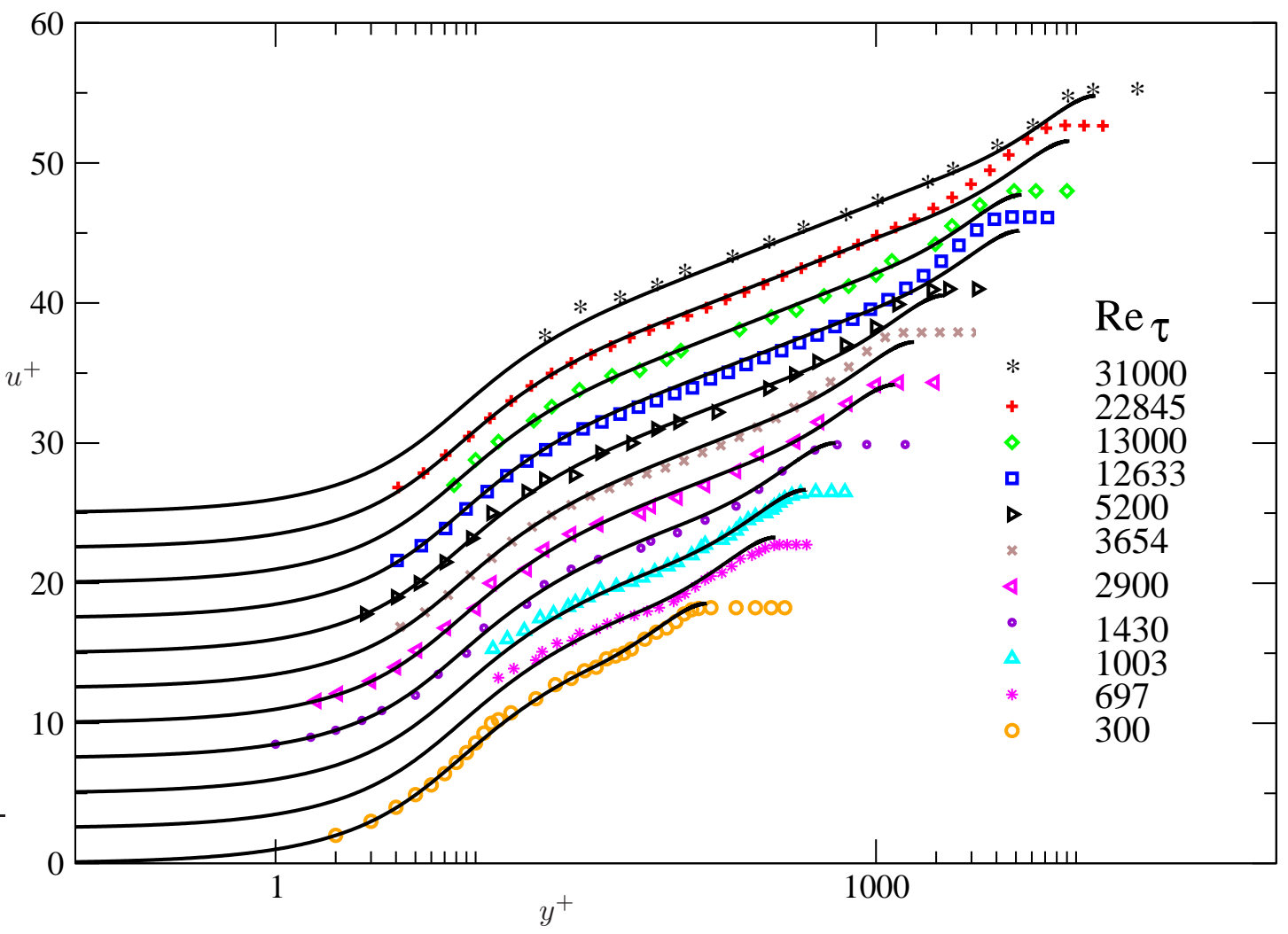

Figure 3. Turbulent boundary layer profiles fitted by the complementary expansion method. Symbols as in table 1 .

The difference between the normalized free-stream velocity from experiment and from the successive complementary expansion method is further investigated in figure 4, where the outer layer portion of the predicted velocity profile at $R e_{\theta}=22845$ is re-plotted on a larger scale. The continuous black line is the numerical prediction obtained by matching the experimental value of $R e_{\theta}$ in the matched complementary expansion, the red dash-dot line is obtained by matching the experimental value of $R e_{\tau}$ and the dashed blue line shows the predicted profile with a matched normalized free-stream velocity $u_{e}^{+}$. Matching the experimental Reynolds numbers seems to give similar profiles irrespective of whether the target Reynolds number is defined with respect to the outer scaling variables $u_{e} \theta / \nu_{l}$ or the inner scaling variables $u_{\tau} \delta / \nu_{l}$. Fitting the outer profile by imposing the normalized freestream velocity $u_{e}^{+}$seems to over-predict the boundary

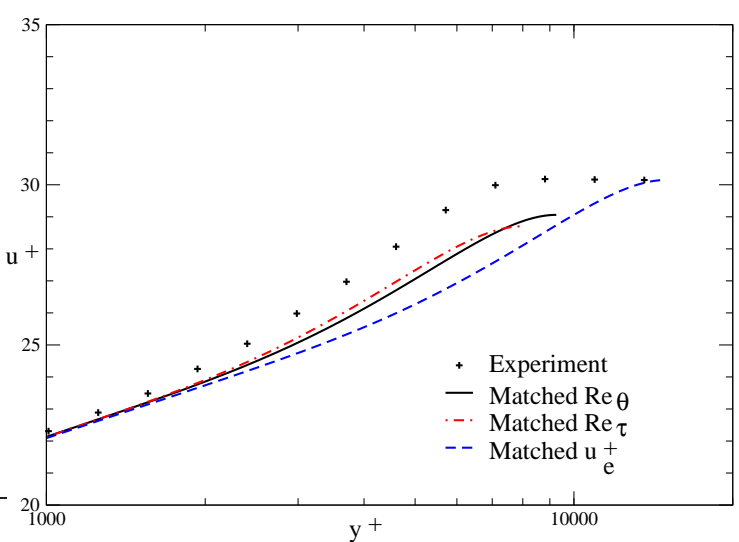

Figure 4. Outer layer profile fitted by the complementary expansion method. $R e_{\theta}=22845 .(+)$ experiment, (-) successive complementary expansion method. layer thickness, leading to a coarser agreement with experiment compared to the numerical predictions obtained by matching the profile Reynolds number.

Figure $5(\mathrm{a})$ compares the normalised mixing length distribution across a zero-pressure gradient boundary layer with $\ell(\eta)$ obtained from measurements at $R e_{\tau}=1540$ by Klebanoff, ${ }^{16}$ reported in Hinze. ${ }^{17}$ The $\ell(\eta)$ distribution from equation 11 is shown by the continuous line while the dashed line shows the distribution from equation 12 with $n=4$. At this Reynolds number, there appears to be a good improvement in the predicted mixing length using the new formulation. No effort has been made to further optimize $n \in \Re$ by adding decimal digits. 
Figure 5(b) shows the profile of the normalized eddy viscosity $\nu_{t} /\left(u_{\tau} F_{1} \delta\right)$ across the same zero pressure gradient boundary layer of figure $5(\mathrm{a})$, where $\nu_{t}=\tilde{\mathrm{F}}^{2} \ell|\partial u / \partial y|$. The symbols are from the same experiment ${ }^{16}$ as in figure 5(a) (open circles) to which further measurements by Townsend ${ }^{18}$ at $R e_{\tau}=2775$ have been added (open squares). Using the mixing length model of Michel et al., ${ }^{10}$ eq. 11, under-predicts the eddy viscosity, as shown by the continuous line, whereas a better fit is achieved by using eq. 12. As a numerical experiment, the target Reynolds number in the successive complementary expansion method was varied over the range $1000 \leq R e_{\tau} \leq 2775$ and was found to have very little effect on the predicted normalized $\nu_{t}$, which is also the trend in experiment. ${ }^{16,18}$

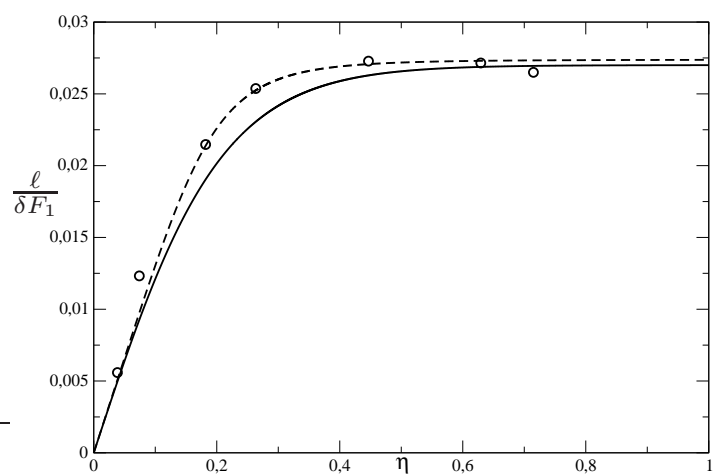

(a) Normalised mixing length $\ell /\left(\delta F_{1}\right)$ versus normalized distance from the wall $\eta$ at $R e_{\tau}=1540$.

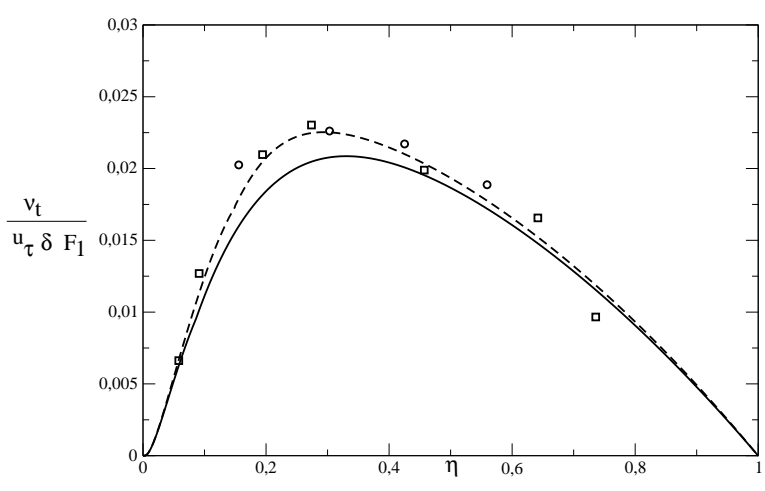

(b) Normalized eddy viscosity $\frac{\nu_{t}}{u_{\tau} \delta F_{1}}$ versus normalized distance from the wall $\eta$.

Figure 5. Turbulence model variables. (o) experiment ${ }^{16}$ at $R e_{\tau}=1540,(\square)$ experiment ${ }^{18}$ at $R e_{\tau}=2775,(--)$ matched complementary expansion at $R e_{\tau}=1000$ with $F_{1}=3.1479$ from eq. 11, (-) matched complementary expansion at $\operatorname{Re}_{\tau}=1000$ with $F_{1}=3.1044$ from eq. 12 .

This paper has not attempted to predict the time-averaged velocity profiles of boundary layers at $R e_{\tau}<$ 300 using the matched complementary expansion method. In this method, $u_{e}^{+}$is obtained by matching the outer region velocity profile to the inner region velocity profile in the logarithmic layer. At $R e_{\tau}<140$, an overlap region in the form of a logarithmic layer is no longer present, which prevents the method form evaluating $u_{e}^{+}$. Here the matched complementary expansion method in its present formulation has reached its lower $R e_{\tau}$ applicability limit.

\section{Favourable pressure gradient boundary layers}

\section{V.A. Sink flow}

Consider a two-dimensional potential sink of strength $Q$ located at the trailing edge of a two-dimensional flat plate, as sketched in figure 6 . The sink induces a streamwise velocity $u_{0}$ at the leading edge of the plate, at $x=0$, from which an incompressible turbulent boundary layer develops along the plate length $L$. It is assumed that the favourable pressure gradient induced by the potential sink of

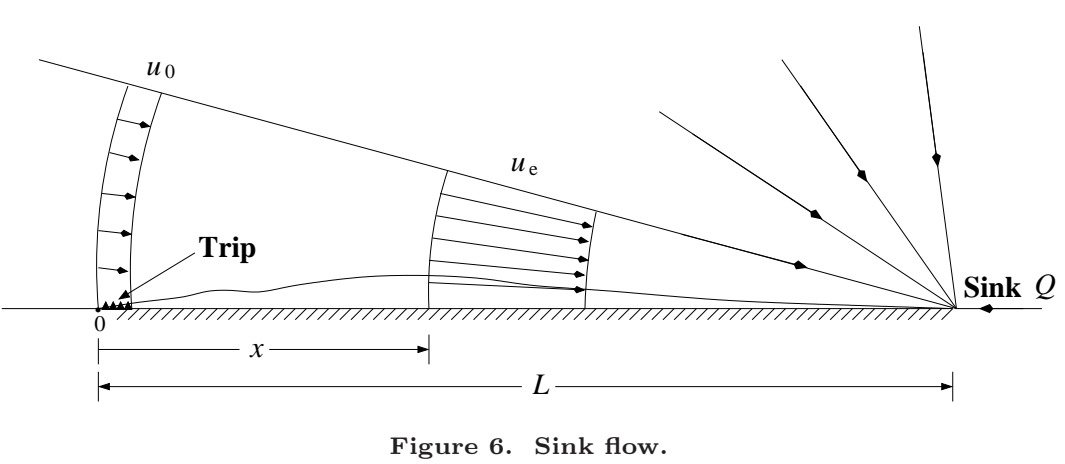
strength $Q$ does not re-laminarize the boundary layer that remains turbulent over the full length $L$ of the plate. Neglecting boundary layer displacement thickness effects, from mass conservation, the local time-averaged free-stream velocity $u_{e}$ is given by

$$
\frac{u_{e}}{u_{0}}=\frac{1}{1-x / L}
$$


The sink flow is uniquely characterized by the positive constant acceleration parameter

$$
K=\frac{\nu_{l}}{u_{e}^{2}} \frac{\mathrm{d} u_{e}}{\mathrm{~d} x}=\frac{\nu_{l}}{u_{0} L} .
$$

The sink flow is a smooth wall boundary layer that satisfies the conditions for "precise equilibrium", ${ }^{19-21}$ in that the mean defect velocity profile and the Reynolds stress profile are invariant with the streamwise coordinate $x$. Coles ${ }^{6}$ proposed that the sink flow at equilibrium gives a pure wall flow velocity profile, eq. 8 , in which $\Pi=0$. He provided several arguments to support his hypothesis but no rigorous proof was given that $\Pi=0$ corresponds to sink flow at equilibrium. This work is presented on the premise that Coles' hypothesis is acceptable.

Perry et al. ${ }^{22}$ derived a formula for the total shear stress distribution along the flow-normal direction by combining the law of the wake with the mean continuity and mean momentum equations:

$$
\frac{\tau}{\tau_{0}}=f_{1}\left(\eta, \Pi, u_{e}^{+}\right)+g_{1}\left(\eta, \Pi, u_{e}^{+}\right) \beta_{c}
$$

where $\beta_{c}=\left(\delta^{*} / \tau_{0}\right) \mathrm{d} \Pi / \mathrm{d} x$ is the Clauser parameter. ${ }^{23}$ The explicit forms of $f_{1}$ and $g_{1}$ are given in Perry et al. $^{22}$ and depend only on the law of the wake. From the momentum integral equation and using the momentum and the displacement thicknesses, that depend on the wake function, the evolution equations are obtained

$$
\begin{array}{r}
F_{1}\left(u_{e}^{+}\right)^{2} E(\Pi) \exp \left(\kappa u_{e}^{+}\right) K+\beta_{c}=0 \\
E(\Pi) \exp \left(\kappa u_{e}^{+}\right) \frac{u_{e}^{+}}{\lambda} \frac{\mathrm{d} u_{e}^{+}}{\mathrm{d} R_{x}}=R\left(\Pi, u_{e}^{+}, \beta_{c}\right)
\end{array}
$$

where $\lambda=u_{e} / u_{0}, R_{x}=x u_{0} / \nu_{l}, E(\Pi)$ and $R\left(\Pi, u_{e}^{+}, \beta_{c}\right)$ are detailed in Perry et al. $^{22}$

Perry et al. ${ }^{22}$ matched the shear stress distribution along $\eta=0.4$ of a quasi-equilibrium boundary layer sink flow to obtain

$$
\beta_{c}=\frac{-f_{1}\left(0.4, \Pi, u_{e}^{+}\right)+f_{1}(0.4, \Pi, \infty)}{g_{1}\left(0.4, \Pi, u_{e}^{+}\right)}+\frac{g_{1}(0.4, \Pi, \infty)}{g_{1}\left(0.4, \Pi, u_{e}^{+}\right)} \beta_{a e}(\Pi)
$$

where $\beta_{a e}(\Pi)$ is the limit value of eq. 32 for $u_{e}^{+} \rightarrow \infty .^{24}$ Green et al. ${ }^{25}$ proposed:

$$
\beta_{a e}=0.03\left[\frac{C_{2}(\Pi)}{F_{1}}\right]^{2}-1.25,
$$

where $C_{2}(\Pi)=\int_{0}^{1} F^{\prime 2}(\eta) \mathrm{d} \eta$. Jones et al. ${ }^{21}$ proposed an empiric formula for $\beta_{a e}$,

$$
\beta_{a e}=-0.5+1.38 \Pi+0.13 \Pi^{2}
$$

based on curve fitting over the range $0 \leq \Pi<0.4$.

Equation 30 can be solved numerically to obtain $\Pi\left(u_{e}^{+}\right)$. Substituting this relation into eq. 31 and setting the initial condition $u_{0}^{+}=19$ at $x=0$, that corresponds to a freshly tripped turbulent boundary layer, eq. 31 can be solved to give

$$
\begin{array}{r}
x / L=1-\exp \left[-K \int_{u_{0}^{+}}^{u_{e}^{+}} H\left(u_{e}^{+}\right) \mathrm{d} u_{e}^{+}\right], \\
H\left(u_{e}^{+}\right)=\frac{u_{e}^{+} E\left[\Pi\left(u_{e}^{+}\right)\right] \exp \left(\kappa u_{e}^{+}\right)}{R\left\{\Pi\left(u_{e}^{+}\right), u_{e}^{+}, \beta_{c}\left[\Pi\left(u_{e}^{+}\right), u_{e}^{+}\right]\right\}} .
\end{array}
$$

Perry et al. ${ }^{26}$ generalized eq. 35 to include the effect of the wake strength gradient for non-equilibrium boundary layers by solving an ordinary system of differential equations.

Equation 35 describes implicitly the streamwise variation of the wall shear stress along the flat plate. This relationship is used in subsection V.B to compare the streamwise variation of the normalized shear stress $u_{e}^{+}$and of other boundary layer integral parameters between theory and experiment. 


\section{V.B. Sink flow results}

In this subsection, the law of the wake of eq. 8 is used to predict the streamwise variation of the integral parameters characterizing the sink flow boundary layer of subsection V.A. This exercise follows that of Perry et al. ${ }^{22}$ where the laws of the wake of Coles ${ }^{6}$ and Jones et al. ${ }^{21}$ were used. The aim is to show that there is some improvement in the prediction of these non-dimensional parameters by using eq. 8 in place of the laws of the wake in the literature ${ }^{6,21}$ and that the analytical method presented herein is performing at least as well as the literature benchmark in predicting a pure wake flow.

Figures 7(a) and 7(b) show the changes in the non-dimensional parameters $\Pi$ and $u_{e}^{+}$in the streamwise direction, as determined by solving eq. 35 using eqs. 33 and 8 (black curves) and eqs. 33 and the law of the wake by Jones et al. ${ }^{21}$ (red curves). The starting condition is $u_{0}^{+}=19$ at $x=0$ that corresponds to a turbulent boundary layer tripped at the leading edge, as sketched in figure 6 . The numerical results of eq. 35 are obtained at the three different values of the acceleration parameter, $K=2.7 \times 10^{-6}$ (solid curve), $K=3.56 \times 10^{-6}$ (dash-dot curve), and $K=5.26 \times 10^{-6}$ (dashed curve). In figs. 7 (a) and 7(b), these predictions are compared against experimental values from Jones et al. ${ }^{21}$ at $K=2.7 \times 10^{-6}(*)$, at $K=3.56 \times 10^{-6}(+)$, and at $K=5.26 \times 10^{-6}(\triangle)$. Using the law of the wall of Jones et al., eq. 35 results in a distribution of $\Pi$ (red curves) significantly higher than in experiment (symbols). The over-prediction from using the law of the wall of Jones et al. (red curves) is partially recovered by using eq. 8 that introduces a vertical shift of $\Pi \approx-1 / 6$ in the $\Pi$ vs $u_{e}^{+}$plot. This results in the black curves that are identical in shape to the red curves but are closer to the experimental values, due to the $\Pi \approx-1 / 6$ vertical shift.

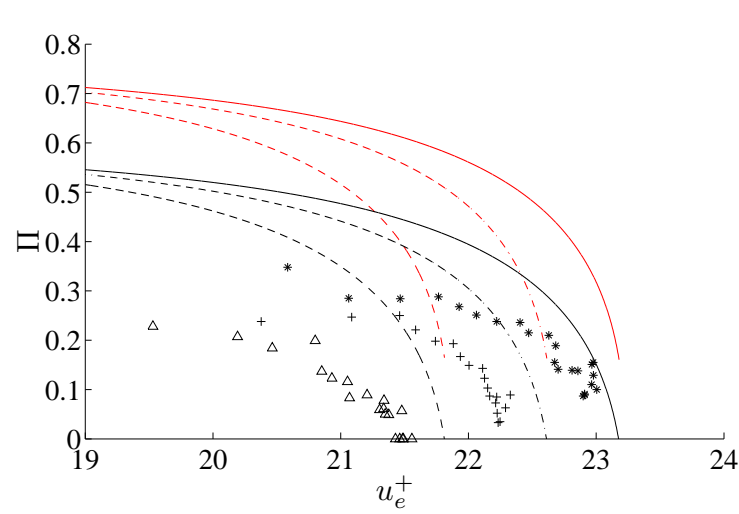

(a) Variation of the wake parameter $\Pi$ with the normalized free-stream velocity $u_{e}^{+}$.

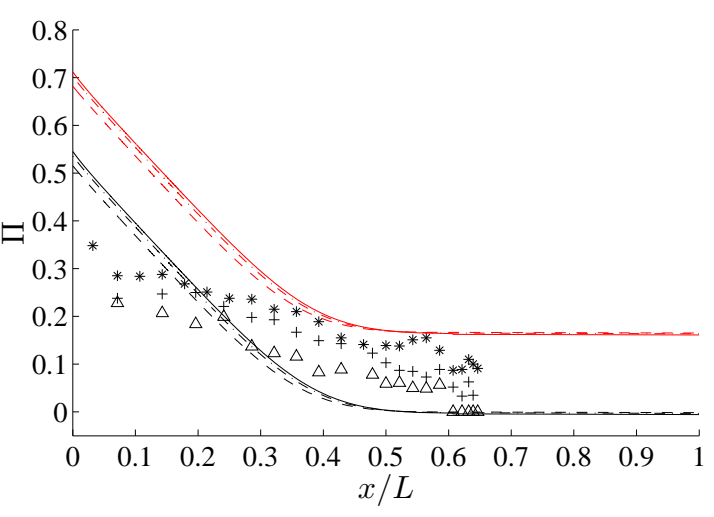

(b) Streamwise variation of the wake parameter $\Pi$.

Figure 7. Variation of the wake parameter $\Pi$ in a sink flow. $\beta_{a e}$ from eq. 33.

Figure 7(b) shows the variation of the wake parameter $\Pi$ in the streamwise direction. The physical significance of the wake parameter is to allow for the presence of a wake region at the top of a turbulent boundary layer in which the velocity profile departs from being a pure wall flow. The acceleration due to the potential sink is such to stop the growth of the wake region, so that close to the sink the wake region thickness approaches zero. This implies that the proper asymptotic value of $\Pi$ close to the potential sink is 0 . Using law of the wall by Jones et al. and eq. 33 results in $\Pi \approx 1 / 6$ as $x / L \rightarrow 1$, as shown by the red curves in figure 7(b). The correct asymptotic behavior of $\Pi \rightarrow 0$ as $x / L \rightarrow 1$ is recovered when eq. 8 and eq. 33 are used, as shown by the black curves in fig. 7(b). This indicates that the law of the wake of equation 8 is an improvement over the formulation by Jones et al. in predicting the asymptotic wake region of a turbulent boundary layer with a strong favourable pressure gradient.

The variation of $\operatorname{Re}_{\theta}$ and of $u_{0} \delta / \nu_{l}$ with $x / L$ using eq. 33 and eq. 8 is identical to the one given in Jones et al. ${ }^{21}$ where the law of the wake by Jones et al. ${ }^{21}$ was used, and is therefore not reported here.

Figures $8(\mathrm{a})$ to 9 (b) show the streamwise variation of the non-dimensional parameters obtained using the empirical formula of eq. 34 in place of eq. 33. In these figures, the curves show predictions from solving eq. 35. The red curves are obtained using the law of the wake by Jones et al. ${ }^{21}$ and eq. 34 and the black curves are obtained using eqs. 8 and 34. The same line patterns (solid, dashed, dash-dot) as in fig. 7(a) are used to identify respectively the same three values of the acceleration parameter $K$. The symbols are experimental values from Jones et al. ${ }^{21}$ and are in the same notation as in fig. 7(a).

Figure 8 (a) shows that, at the lowest value of the acceleration parameter $K=2.7 \times 10^{-6}$, there is a 


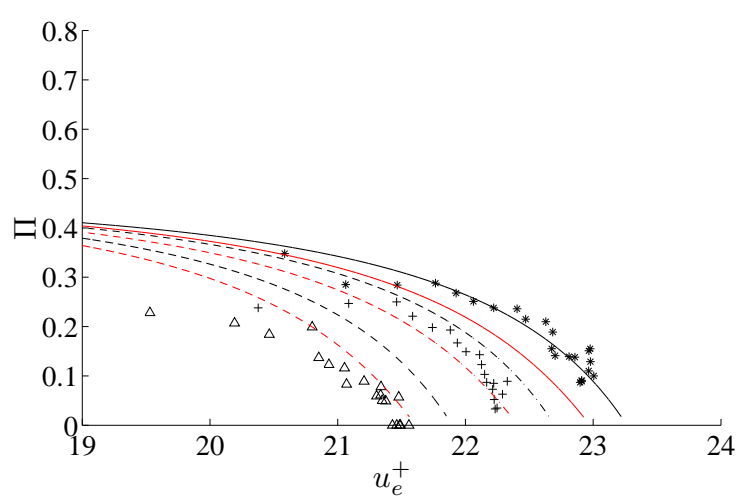

(a) Variation of the wake parameter $\Pi$ with the normalized free-stream velocity $u_{e}^{+}$.

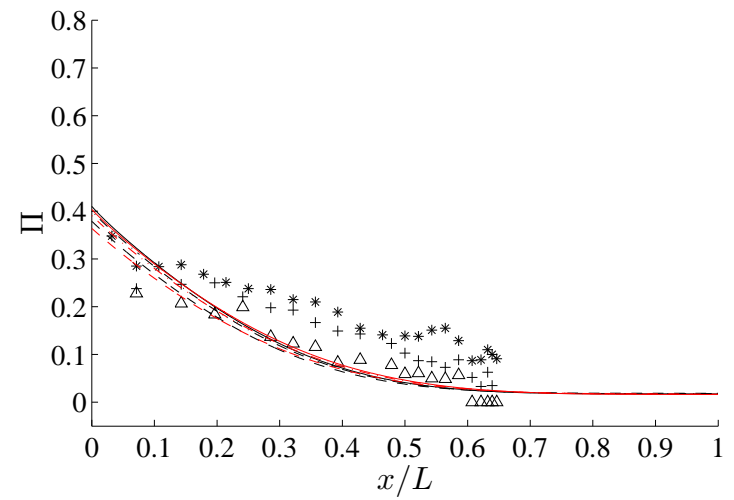

(b) Streamwise variation of the wake parameter $\Pi$.

Figure 8. Variation of the wake parameter $\Pi$ in a sink flow. $\beta_{a e}$ from eq. 34 .

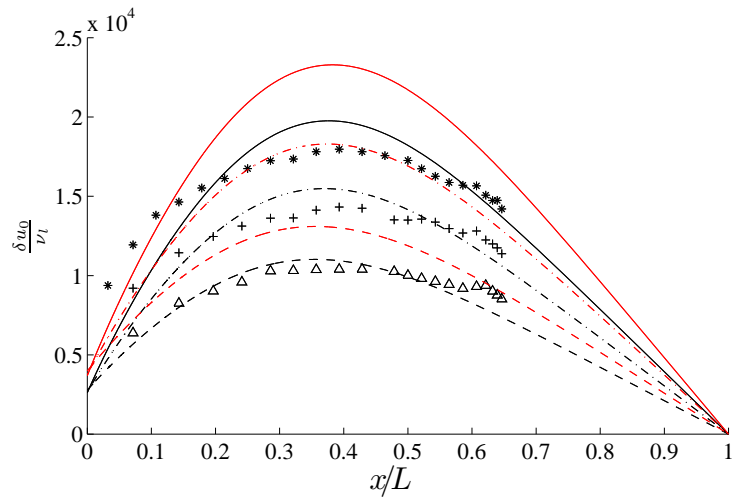

(a) Streamwise variation of $\delta u_{0} / \nu_{l}$.

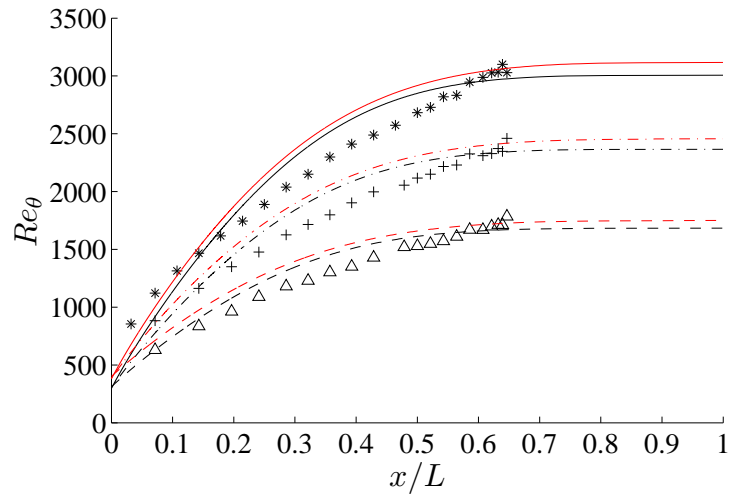

(b) Streamwise variation of $R e_{\theta}$.

Figure 9. Streamwise variation of sink flow parameters. $\beta_{a e}$ from eq. 34 . 
significant improvement in the prediction of the variation of $\Pi$ with $u_{e}^{+}$by using eq. 8 . The black curve fits through the cloud of experimental data $(*)$ better than the red curve, which under-estimates $\Pi$ at this value of $K$. At the higher acceleration parameter $K=3.56 \times 10^{-6}$, the dash-dot curves from the law of the wake by Jones et al. ${ }^{21}$ and eq. 8 lie respectively below and above the experimental data $(+)$, with neither equation giving a significantly better prediction than the other. At the highest value of the acceleration parameter $K=5.26 \times 10^{-6}$, neither equation gives a good fit to the experimental data, although the red dashed line from the law of the wake by Jones et al. ${ }^{21}$ is the closest.

Figure 8(b) shows the streamwise variation of the wake parameter $\Pi$ predicted using eq. 34 . While in eq. $33 \beta_{a e}$ depends directly on the boundary layer shape $\eta$, eq. 34 is an empirical regression with constant coefficients, invariant on the profile shape. Therefore the streamwise distribution of $\Pi$ in figure $8(\mathrm{~b})$ is also insensitive to changes in the law of the wake, resulting in predictions that essentially overlap for all values of $K$. The predictions are an improved fit to the experimental data with respect to figure $7(\mathrm{~b})$, which reflects the regression used to obtain eq. 34. The asymptotic trend of $\Pi \rightarrow 0$ as $x / L \rightarrow 1$ is captured less well than with eqs. 8 and 33, black curves in figure $7(\mathrm{~b})$, possibly due to the lack of experimental values at $x / L \rightarrow 1$ on which eq. 34 is regressed.

Figure 9(a) shows the streamwise variation of the normalized boundary layer thickness at different values of the acceleration parameter $K$. The symbols are experimental values from Jones et al. ${ }^{21}$ taken at the same values of $K$ as in figure 8(b). These data are labelled as in fig. 8(a). Predictions of $\delta u_{0} / \nu_{l}$ are obtained using eq. 8 (black curves) and using the law of the wake by Jones et al. ${ }^{21}$ (red curves). The best match between prediction and experiment is obtained by using eq. 8 at all $K$. This is because in deriving eq. 8 the height of the boundary layer $\delta$ is used as one of the closure parameters, whereas the law of the wake by Jones et al. ${ }^{21}$ satisfies eq. 6 but not eq. 7 , leading to the coarser match in the streamwise variation of the normalized boundary layer thickness shown in figure $9(\mathrm{a})$.

Figure 9(b) shows the streamwise variation of the momentum thickness based Reynolds number approaching the sink located at $x=L$. The same notation as fig. 9 (a) is used. $R e_{\theta}$ grows monotonically in the downstream direction, with a reducing rate at increasing values of the acceleration parameter $K$. Approaching the sink, the flow acceleration reduces the growth rate to zero, so that $R e_{\theta}$ asymptotes to a constant value that is $K$ dependent. The predictions of $R e_{\theta}$ appear to be weakly dependent upon whether the law of the wake by Jones et al. ${ }^{21}$ or eq. 8 are used for their estimate. The relative insensitivity of the momentum thickness Reynolds number on the wake function agrees with the findings from using eq. 33 and eq. 8 , the predictions from which are identical to those given in Jones et al. ${ }^{21}$

Comparative predictions of the boundary layer parameters approaching the sink flow from the matched complementary expansion method are not given due to the difficulty of finding a solution at the same values of the acceleration parameter $K$ as in experiment. The acceleration reduces the thickness of the outer layer, so that the method fails to find a match between the inner and the outer layer velocity profile that is continuous in $u^{+}$and in $d u^{+} / d y^{+}$across the interface. This outcome is in-line with the sink flow satisfying the conditions for "precise equilibrium" and giving a pure wall flow velocity profile, which is discontinuous at $\eta=1$. A preliminary analysis of the parameter space for $K$ for which a solution by the matched complementary expansion is available suggests that the method works for $K<10^{-6}$.

\section{Conclusions}

Numerical and analytical methods for obtaining the time-mean velocity profiles of a turbulent boundary layer are presented and validated against experimental data.

The analytical method is an extension to the law of the wake by Coles ${ }^{6}$ that matches both the free stream velocity and the velocity gradient at the boundary layer edge. The method is shown to predict the outer region of turbulent boundary layers rather well for zero streamwise pressure gradient test cases over the Reynolds number range $300 \leq R e_{\theta} \leq 31000$, with a maximum mean square percentage error of $2.05 \%$.

The authors propose a modification to the successive complementary expansion numerical method in Cousteix \& Mauss, ${ }^{7}$ with a new blending function for the mixing length in the outer region. Comparison against experimental data shows that the new blending function improves the prediction of the mixing length and of the eddy viscosity in outer region of a zero pressure gradient boundary layer. The new method is validated against experimental velocity profile data over the Reynolds number range $300 \leq R e_{\theta} \leq 31000$ under zero streamwise pressure gradient and found to achieve engineering accurate predictions. The new blending function introduces an additional adjustable parameter $n \in \Re$ in the model that can undergo a 
more extensive calibration over a wider experimental dataset to further improve the predictions.

A sink flow test case was used to study the change in the wake region thickness in an accelerating flow. By using the new law of the wake, the correct asymptotic behavior for the wake parameter $\Pi$ is recovered, namely $\Pi \rightarrow 0$ at $x / L \rightarrow 1$, when the streamwise variation of $\Pi$ is evaluated from the integral length scales $F_{1}$ and $C_{2}$ of the defect law under quasi-equilibrium conditions.

Using a parabolic distribution for the Clauser parameter at equilibrium, $\beta_{a e}$, independent from $F_{1}$ and $C_{2}$, reduces the sensitivity of the predictions on the choice of the wake function. The new law of the wake improves to some extent the predictions of the non-dimensional boundary layer thickness distribution.

\section{Acknowledgments}

This research project has been supported by a Marie Curie Research Training Fellowship of the European Community's Sixth Framework Programme under contract MEST CT 2005 020301. This paper was written with the support of the Invited Professorship scheme of the Université Paul Sabatier, Toulouse.

\section{References}

${ }^{1}$ Rona, A. and Brooksbank, E. J., "Injection parameters for an effective passive control of the cavity flow instability," 40th AIAA Aerospace Sciences Meeting 83 Exhibit, AIAA, Reno, NV, Jan. 2002.

${ }^{2}$ Grottadaurea, M. and Rona, A., "Noise sources from a cylindrical cavity," 13th AIAA/CEAS Aeroacoustics Conference, No. 2007-3723, AIAA, Rome, Italy, May 2007.

${ }^{3}$ Grottadaurea, M. and Rona, A., "The role of the inflow momentum thickness in subsonic cylindrical cavity noise generation," 14th International Congress on Sound and Vibration, No. 165, International Institute of Acoustics and Vibration, Cairns, Australia, July 2007.

${ }^{4}$ Rowley, C., Colonius, T., and Basu, A., "On Self-sustained Oscillations in Two-dimensional Compressible Flow Over Rectangular Cavities," J. Fluid Mechanics, Vol. 455, 2002, pp. 315-46.

${ }^{5}$ Wilcox, D., Turbulence modeling for CFD, D. C. W. Industries, 2nd ed., December 2002.

${ }^{6}$ Coles, D., "The law of the wake in the turbulent boundary layer," J. Fluid Mech., Vol. 1, January 1956, pp. 191-226.

${ }^{7}$ Cousteix, J. and Mauss, J., Asymptotic analysis and boundary layers, Springer-Verlag, Berlin Heidelberg, 2007.

${ }^{8}$ Prandtl, L., "Bericht über Untersuchungen zur ausgebildeten Turbulenz," Z. Angew. Math. Mech., Vol. 5, 1925, pp. 136139 .

${ }^{9}$ Van Driest, E. R., "On dimensional analysis and the presentation of data in fluid-flow problems," ASME Journal of Applied Mathematics, Vol. 13, No. 1, March 1946, pp. 34-40.

${ }^{10}$ Michel, R., Quémard, C., and Durant, R., "Application d'un schéma de longueur de mélange à l'étude des couches limites turbulentes d'équilibre," Technical Note 154, ONERA, 1969.

${ }^{11}$ Cousteix, J., "Outer boundary layer self-similar solution," Private communication.

${ }^{12}$ Spalart, P. R., "Direct simulation of a turbulent boundary layer up to $R_{\theta}=1410$," J. Fluid Mech., Vol. 187, May 1988, pp. 61-98.

${ }^{13}$ Erm, L. and Joubert, P. N., "Low-Reynolds-number turbulent boundary layers," J. Fluid Mech., Vol. 230, Jan. 1991, pp. 1-44.

${ }^{14}$ De Graaff, D. B. and Eaton, J. K., "Reynolds-number scaling of the flat-plate turbulent boundary layer," J. Fluid Mech., Vol. 442, June 2000, pp. 319-346.

${ }^{15}$ Österlund, J. M., Experimental studies of zero pressure-gradient turbulent boundary layer flow, Phd thesis, Royal Inst. Techn., KTM, Stockholm, 1999.

${ }^{16}$ Klebanoff, P. S., "Characteristics of turbulence in a boundary layer with zero pressure gradient," Technical Note 3178, National Advisory Committee for Aeronautics (NACA), 1954.

${ }^{17}$ Hinze, J. O., Turbulence, McGraw-Hill, New York, 1975

${ }^{18}$ Townsend, A. A., "The structure of the turbulent boundary layer," Mathematical Proceedings of the Cambridge Philosophical Society, Vol. 47, No. 2, April 1951, pp. 375-395.

${ }^{19}$ Townsend, A. A., "Equilibrium layers and wall turbulence," J. Fluid Mech., Vol. 11, Aug. 1961, pp. 97-120.

${ }^{20}$ Rotta, J. C., "Turbulent boundary layers in incompressible flow," Prog. Aero. Sci., Vol. 2, 1962, pp. 1-219.

${ }^{21}$ Jones, M. B., Marušić, I., and Perry, A. E., "Evolution and structure of sink-flow turbulent boundary layers," J. Fluid Mech., Vol. 428, June 2001, pp. 1-27.

${ }^{22}$ Perry, A. E., Marušić, I., and Li, J. D., "Wall turbulence closure based on classical similarity laws and the attached eddy hypothesis," Phys. Fluids, Vol. 6, No. 2, Feb. 1994, pp. 1024-1035.

${ }^{23}$ Clauser, F., "The turbulent boundary layer," Adv. Appl. Mech., Vol. 4, 1956, pp. 1-51. 1992.

${ }^{24}$ Perry, A., "A new look at some closure problems of turbulent boundary layers," Tech. rep., GALCIT, Cal. Inst. Techn.,

${ }^{25}$ Green, J. E., Weeks, D. J., and Brooman, J. W. F., "Prediction of turbulent boundary layers and wakes in compressible flow by lag-entrainment method," Rep. and Mem. 3791, Aero. Res. Coun., London, UK, 1973.

${ }^{26}$ Perry, A. E., Marušić, I., and Jones, M. B., "On the streamwise evolution of turbulent boundary layers in arbitrary pressure gradients," J. Fluid Mech., Vol. 461, Jan. 2002, pp. 61-91. 